\title{
AN APPROXIMATION THEOREM FOR SEQUENCES OF LINEAR STRAINS AND ITS APPLICATIONS
}

\author{
KEWEI ZHANG ${ }^{1}$
}

\begin{abstract}
We establish an approximation theorem for a sequence of linear elastic strains approaching a compact set in $L^{1}$ by the sequence of linear strains of mapping bounded in Sobolev space $W^{1, p}$. We apply this result to establish equalities for semiconvex envelopes for functions defined on linear strains via a construction of quasiconvex functions with linear growth.
\end{abstract}

Mathematics Subject Classification. 26B25, 41A30, 49J45.

Received July 4, 2002. Revised September 22, 2003.

\section{INTRODUCTION AND MAIN RESULTS}

This paper establishes an approximation theorem for sequences of linear elastic strains approaching a compact set in the space of symmetric matrices. We apply the result to the study of equality of various semiconvex envelopes for functions defined on the space of linear strains. We show that under a simple coercivity condition, $Q_{e}(f)=C(f)$ if and only if $R_{e}(f)=C(f)$, where $Q_{e}(f)$ and $R_{e}(f)$ are the quasiconvex and rank-one convex envelopes of $f$ on linear strains respectively. Before we state our main results, let us introduce some notation.

For $A \in M^{n \times n}$ - the space of $n \times n$ real matrices with the standard Euclidean inner product on $\mathbb{R}^{n^{2}}$, we let $e(A)=\left(A+A^{T}\right) / 2$, where $A^{T}$ is the transpose of $A$. If $u$ is a smooth mapping from a domain $\Omega \subset \mathbb{R}^{n}$ to $\mathbb{R}^{n}$, we call $e(D u(x))$ the linear elastic strain of $u$ where $D u$ is the gradient of $u$. Let $M_{s}^{n}$ and $\left(M_{s}^{n}\right)^{\perp}$ be the subspaces of symmetric and skew-symmetric matrices in $M^{n \times n}$ respectively, we see that $e(A)=P_{M_{s}^{n}}(A)$, where $P_{M_{s}^{n}}$ is the orthogonal projection from $M^{n \times n}$ onto $M_{s}^{n}$. Let $\operatorname{dist}(Y, K)=\inf _{X \in K}|Y-X|$ be the distance function from $Y \in M_{s}^{n}$ to a closed set $K \subset M_{s}^{n}$. The following is our approximation theorem.

Theroem 1. Let $\Omega \subset \mathbb{R}^{n}$ be a Lipschitz domain and $u_{j} \in C_{0}^{\infty}\left(\Omega, \mathbb{R}^{n}\right)$ such that

$$
\lim _{j \rightarrow \infty} \int_{\Omega} \operatorname{dist}\left(e\left(D u_{j}(x)\right), \bar{B}_{R}(0)\right) \mathrm{d} x=0,
$$

where $\bar{B}_{R}(0)$ is the closed ball in $M_{s}^{n}$ centered at 0 with radius $R$. Then there is a subsequence $\left(u_{j_{k}}\right)$ of $\left(u_{j}\right)$, and a sequence of Lipschitz mappings $v_{k}: \mathbb{R}^{n} \rightarrow \mathbb{R}^{n}$ such that for every $1<p<\infty$,

$$
\int_{\mathbb{R}^{n}}\left|e\left(D v_{k}\right)\right|^{p} \mathrm{~d} x \leq C(n, p)<+\infty, \quad \text { and } \lim _{k \rightarrow \infty} \int_{\Omega}\left|e\left(D u_{j_{k}}\right)-e\left(D v_{k}\right)\right| \mathrm{d} x=0 .
$$

Keywords and phrases. Linear strains, maximal function, approximate sequences, quasiconvex envelope, quasiconvex hull.

1 School of Mathematical Sciences, University of Sussex Brighton, BN1 9QH, UK; e-mail: k.zhang@sussex.ac.uk 
Note to Theorem 1. As kindly pointed to me by I. Fonseca and the referee, there is an alternative proof of Theorem 1 by using $A$-qausiconvexity. Further details can be found in the remark after the proof of Theorem 1 .

Corollary 1. Let $\left(v_{k}\right)$ be the sequence of Lipschitz mappings given by Theorem 1 . Then for each $1<p<\infty$, there are a skew-symmetric matrix $A(p)_{k} \in\left(M_{s}^{n}\right)^{\perp}$ and some $x_{k} \in \mathbb{R}^{n}$ satisfying $\int_{\Omega} A(p)_{k}\left(x-x_{k}\right) \mathrm{d} x=0$ such that the sequence $w_{k}: \Omega \rightarrow \mathbb{R}^{n}$ defined by

$$
w_{k}(x)=v_{k}-\frac{1}{\operatorname{meas}(\Omega)} \int_{\Omega} v_{k}(y) \mathrm{d} y-A_{k}(p)\left(x-x_{k}\right), x \in \Omega
$$

is bounded in $W^{1, p}\left(\Omega, \mathbb{R}^{n}\right)$,

$$
\int_{\Omega}\left|D w_{k}\right|^{p} \mathrm{~d} x \leq C \int_{\Omega}\left|e\left(D w_{k}\right)\right|^{p} \mathrm{~d} x \leq C_{0}(p)<+\infty, \quad \int_{\Omega} w_{k} \mathrm{~d} x=0,
$$

where $C, C_{0}(p)$ are positive constants independent of $k$ and

$$
\lim _{k \rightarrow \infty} \int_{\Omega}\left|e\left(D u_{j_{k}}\right)-e\left(D w_{k}\right)\right| \mathrm{d} x=0
$$

In Theorem 1 , the ball $\bar{B}_{R}(0)$ can be replaced by any compact set $K \subset M_{s}^{n}$. Theorem 1 and Corollary 1 are motivated from an approximation result in [38] for $W_{0}^{1,1}$ approximating sequences of gradients approaching a compact set in $M^{N \times n}$ by a bounded $W^{1, \infty}$ sequence. The statements of Theorem 1 and Corollary 1 are almost optimal in the sense that they are false if $p=+\infty$ [24]. The main tool for establishing Theorem 1 is a generalized version of Liu's Luzin type theorem [25] to the space of bounded deformations $B D(\Omega)[12]$. Combining the result in [12] with some classical estimates for standard singular integral operators [30] enables us to prove Theorem 1. Corollary 1 then follows from Poincaré's inequality and Korn's inequality [20].

The following is our main application of Theorem 1 and Corollary 1 to equalities of some semiconvex envelopes for functions defined on linear strains. The study of quasiconvex functions defined on linear elastic strains is closely related to the variational approach to material microstructure by using geometrically linear models $[9,10,18]$. Functions defined on $M_{s}^{n}$ with linear growth are important in the theory of plasticity [3,21,35]. For a continuous function $f: M_{s}^{n} \rightarrow \mathbb{R}$ bounded below, let $Q_{e}(f)$ and $R_{e}(f)$ be the quasiconvex and rank-one convex envelopes of $f$ respectively (see Sect. 2 for definitions). We denote by $C(f)$ the convex envelope of $f$.

Theroem 2. Suppose $f: M_{s}^{n} \rightarrow \mathbb{R}$ is continuous and satisfies, for $A \in M_{s}^{n}$

$$
\lim _{|A| \rightarrow+\infty} \frac{f(A)}{|A|}=+\infty .
$$

Then $Q_{e}(f)=C(f)$ if and only if $R_{e}(f)=C(f)$.

Theorem 2 was established for functions $f: M^{N \times n} \rightarrow \mathbb{R}[43]$ under the coercivity condition (1.5) for $A \in M^{N \times n}$. The difference between Theorem 2 and that in [43] is that in the present situation, the function $X \rightarrow f(e(X))$ is not coercive in the sense of (1.5) for $X \in M^{n \times n}$. A weaker version of Theorem 2 was proved in [44], via an elementary argument for functions defined on linear strains under the assumption that $f(A) \geq c\left(|A|^{2}-1\right)$.

The following result on the construction of quasiconvex functions on linear strains with linear growth will be used indirectly to establish Theorem 2 through some of its implications. The construction itself is of independent interest and its proof depends on Theorem 1 and Corollary 1.

Theroem 3. Suppose $F: M_{s}^{n} \rightarrow \mathbb{R}$ is quasiconvex on linear strains and is bounded below. Assume that $0 \leq$ $F(Y) \leq C_{0}\left(1+|Y|^{p}\right)$ for $Y \in M_{s}^{n}$, and that for some real $\alpha \geq 0$, the sub-level set $K_{\alpha}:=\left\{Y \in M_{s}^{n}: F(Y) \leq \alpha\right\}$ 
is compact. Then, for every $1 \leq p<+\infty$, the quasiconvex function on linear strains $Q_{e} \operatorname{dist}^{p}\left(A, K_{\alpha}\right), A \in M_{s}^{n}$ satisfies

for $Y \in M_{s}^{n}$ and

$$
C_{0}|Y|^{p}-C_{1} \leq Q_{e} \operatorname{dist}^{p}\left(Y, K_{\alpha}\right) \leq C_{2}\left(1+|Y|^{p}\right)
$$

$$
K_{\alpha}=\left\{Y \in M_{s}^{n}: Q_{e} \operatorname{dist}^{p}\left(Y, K_{\alpha}\right)=0\right\},
$$

where $C_{0}, C_{1}, C_{2}$ are positive constants and $Q_{e} \operatorname{dist}^{p}\left(Y, K_{\alpha}\right)$ is the quasiconvex envelope on linear strains of the p-distance function $\operatorname{dist}^{p}\left(Y, K_{\alpha}\right)$.

We need the following results on various semiconvex hulls of linear strains for compact sets in $M_{s}^{n}$ and their properties to establish Theorem 2. For the $M^{N \times n}$ version of these results, see [33,37,39]. We define two types of quasiconvex hulls on linear strains for closed subsets of $M_{s}^{n}$.

Definition 1.1. Let $K \subset M_{s}^{n}$ be closed, for $1 \leq p<\infty$, we defined the strong $p$-quasiconvex hull $\mathbb{Q}_{p}^{e}(K)$ and weak $p$-quasiconvex hull $Q_{p}^{e}(K)$ respectively as

$$
\begin{gathered}
\mathbb{Q}_{p}^{e}(K)=\left\{X \in M_{s}^{n}, f(A) \leq \sup _{Y \in K} f(Y), f \text { quasiconvex on linear strains }, 0 \leq f(A) \leq C_{f}\left(1+|A|^{p}\right)\right\} \\
Q_{p}^{e}(K)=\left\{A \in M_{s}^{n}, Q_{e} \operatorname{dist}^{p}(A, K)=0\right\}
\end{gathered}
$$

and the strong $p$-rank-one convex hull of linear strains as

$$
\mathbb{R}_{p}^{e}(K)=\left\{X \in M_{s}^{n}, f(A) \leq \sup _{Y \in K} f(Y), f \text { rank-one convex on linear strains }, 0 \leq f(A) \leq C_{f}\left(1+|A|^{p}\right)\right\} .
$$

Clearly, one has $\mathbb{R}_{p}^{e}(K) \subset \mathbb{Q}_{p}^{e}(K) \subset Q_{p}^{e}(K), \mathbb{R}_{p}^{e}(K) \subset \mathbb{R}_{q}^{e}(K)$ and $\mathbb{Q}_{p}^{e}(K) \subset \mathbb{Q}_{q}^{e}(K)$ for $1 \leq q \leq p<\infty$. We can also show that $Q_{p}^{e}(K) \subset Q_{q}^{e}(K)$ (see Proof of Th. 3 and (4.1)).

In order to state Theorem 5 , we need to define the so-called closed lamination convex hull $L_{c}^{e}(K)$ on linear strains for a compact set $K \subset M_{s}^{n}$ as follows [44].

Notice that the subspace $\left(M_{s}^{n}\right)^{\perp}$ of skew-symmetric matrices does not have rank-one matrices. We say that $A \in M_{s}^{n}$ is a compatible linear strain if $\operatorname{span}[A] \oplus\left(M_{s}^{n}\right)^{\perp}$ has rank-one matrices. For example, the identity matrix $I \in M_{s}^{n}$ is incompatible. Two linear strains $A, B \in M_{s}^{n}$ are called compatible if $A-B$ is a compatible linear strain and we call $\{A, B\}$ a compatible pair. Clearly, $A \in M_{s}^{n}$ is compatible if and only if $\{0, A\}$ is a compatible pair. This definition of compatibility is equivalent to that in [22], that is, $A, B \in M_{s}^{n}$ are compatible if either $A-B$ is a rank-one matrix or $A-B$ is of rank two and the two non-zero eigenvalues have opposite signs.

A set $K \subset M_{s}^{n}$ is called lamination convex on linear strains if for every compatible pair $\{A, B\} \subset K$, one has $\{t A+(1-t) B, 0 \leq t \leq 1\} \subset K$. For a compact set $K \subset M_{s}^{n}$, the closed lamination convex hull on linear strains $L_{c}^{e}(K)$ is the smallest closed lamination convex set on linear strains that contains $K$.

The closed laminated convex hull for a compact set $K \subset M^{N \times n}$ was defined in [40] motivated from [28]. It is easy to see that $K \subset L_{c}^{e}(K) \subset \mathbb{R}_{p}^{e}(K) \subset \mathbb{Q}_{p}^{e}(K) \subset C(K)$.

Theroem 4. Let $K \subset M_{s}^{n}$ be non-empty and compact. Then $1 \leq p<\infty$,

$$
\mathbb{Q}_{p}^{e}(K)=Q_{p}^{e}(K)=Q_{1}^{e}(K) .
$$

The following result is an implication of the results established in [44]. For the convenience of the reader, we give a proof in the Appendix.

Theroem 5. Let $K \subset M_{s}^{n}$ be compact. Then $L_{c}^{e}(K)=C(K)$ if and only if $\mathbb{Q}_{2}^{e}(K)=C(K)$. 
From Theorem 4 we see that we may replace $\mathbb{Q}_{2}^{e}$ by any $Q_{p}^{e}$ for $1 \leq p<\infty$. Also $\mathbb{R}_{2}^{e}(K)=C(K)$ if and only if $\mathbb{Q}_{2}^{e}(K)=C(K)$.

In Section 2, we give notation and preliminaries which are needed for establishing our main results. We prove Theorem 1 and Corollary 1 in Section 3. In Section 4 we establish Theorems $2-4$ by applying Theorem 1 directly or indirectly followed by some examples. In the Appendix we give a proof of Theorem 5 and establish an elementary result Lemma 4.1 which is needed in the proof of Theorem 3.

\section{Notation AND PRELiminaries}

Throughout the rest of this paper $\Omega$ denotes a bounded open subset of $\mathbb{R}^{n}$. We denote by $M^{N \times n}$ the space of real $N \times n$ matrices, with norm $|P|=\left(\operatorname{tr} P^{T} P\right)^{1 / 2}$. In this paper we are mainly interested in the case when $N=n \geq 2$. We let $C_{0}^{\infty}\left(\Omega, \mathbb{R}^{n}\right)$ be the space of smooth functions $\phi: \Omega \rightarrow \mathbb{R}^{n}$ having compact support in $\Omega$. We denote the Lebesgue spaces $L^{p}\left(\Omega, \mathbb{R}^{n}\right)$ and Sobolev spaces $W^{1, p}\left(\Omega, \mathbb{R}^{n}\right)$ and $W_{0}^{1, p}\left(\Omega, \mathbb{R}^{n}\right)$ for vector-valued functions $u: \Omega \rightarrow \mathbb{R}^{n}$ as usual [2]. As in Section 1 , we let $M_{s}^{n}$ and $\left(M_{s}^{n}\right)^{\perp}$ be the subspaces of symmetric and skew-symmetric matrices respectively. We see that these two subspaces are orthogonal to each other. For $X \in M^{n \times n}$, we let $e(X)=\left(X+X^{T}\right) / 2=P_{M_{s}^{n}}(X)$ where $P_{M_{s}^{n}}$ is the orthogonal projection from $M^{n \times n}$ to $M_{s}^{n}$. We denote weak convergence of sequences by $\rightarrow$. The Lebesgue measure of a measurable set $S$ in $\mathbb{R}^{n}$ is $\operatorname{meas}(S)$ while the complement of a set $S \subset \mathbb{R}^{n}$ is $S^{c}$. We use various $C$ 's to denote positive constants such as $C(n, p)$. In later sections, two $C(n, p)$ 's in the same line may not be the same. They just mean positive constants depending only on $n$ and $p$.

We define the $p$-distance function from $Y \in M_{s}^{n}$ to a set $K \subset M_{s}^{n}$ by $\operatorname{dist}^{p}(Y, K):=\inf _{A \in K}|Y-A|^{p}$. The following are some results we need later.

Definition 2.1. (See $[4,26]$.) A continuous function $f: M^{N \times n} \rightarrow \mathbb{R}$ is quasiconvex if

$$
\int_{U} f(P+D \phi(x)) \mathrm{d} x \geq f(P) \operatorname{meas}(U)
$$

for every $P \in M^{N \times n}, \phi \in C_{0}^{\infty}\left(U ; \mathbb{R}^{N}\right)$, and every bounded open subset $U \subset \mathbb{R}^{n}$. A function $f: M^{N \times n} \rightarrow \mathbb{R}$ is rank-one convex if for any $A, B \in M^{N \times n}$ with $B$ a rank-one matrix, the function $t \rightarrow f(A+t B)$ is convex.

It is well-known now that quasiconvexity implies rank-one convexity $[4,11,26]$ while the converse is not true [32]. To construct quasiconvex functions, we need the following

Definition 2.2. (See [11].) Suppose $f: M^{N \times n} \rightarrow \mathbb{R}$ is a continuous function. The quasiconvex envelope (rank-one convex envelope, respectively) $Q(f)(R(f)$ respectively) of $f$ is defined by

$$
Q(f)=\sup \{g \leq f ; g \text { quasiconvex }\}, \quad R(f)=\sup \{g \leq f ; g \text { rank-one convex }\}
$$

It is well-known [11] that $C(f) \leq Q(f) \leq R(f) \leq f$ and the quasiconvex envelope $Q f$ can be calculated by

$$
Q f(P)=\inf _{\phi \in C_{0}^{\infty}\left(\Omega ; R^{N}\right)} \frac{1}{\operatorname{meas}(\Omega)} \int_{\Omega} f(P+D \phi(x)) \mathrm{d} x,
$$

where $\Omega \subset \mathbb{R}^{n}$ is a bounded domain. In particular the infimum in (2.1) is independent of the choice of $\Omega$.

For a continuous function $f: M_{s}^{n} \rightarrow \mathbb{R}$, we say that $f$ is quasiconvex (rank-one convex respectively) on linear strains, if the function $F: M^{n \times n} \rightarrow \mathbb{R}$ defined by $F(X)=f(e(X))$ is a quasiconvex (rank-one convex respectively) function. 
We define the quasiconvex envelope $Q_{e}(f)$ and rank-one convex envelope $R_{e}(f)$ on linear strains for a continuous function $f: M_{s}^{n} \rightarrow \mathbb{R}$ by

$$
\begin{aligned}
& Q_{e}(f)=\sup \{g \leq f ; g \text { quasiconvex on linear strains }\} \\
& R_{e}(f)=\sup \{g \leq, f ; g \text { rank-one convex on linear strains }\}
\end{aligned}
$$

The following simple statement is easy to prove.

Proposition 2.1. For a continuous function $f: M_{s}^{n} \rightarrow \mathbb{R}$, let $F(X)=f(e(X))$ for $X \in M^{n \times n}$. Then $Q(F(X))=Q_{e}(f(e(X)))$.

Proof. Clearly $Q_{e}(f(e(X))) \leq Q(F(X))=\sup \{g \leq f ; g$ quasiconvex $\}$. However, if we let $D \subset \mathbb{R}^{n}$ be the unit cube, then

$$
Q(F(X))=\inf _{\phi \in C_{0}^{\infty}\left(D ; \mathbb{R}^{n}\right)} \int_{D} F(X+D \phi) \mathrm{d} x=\inf _{\phi \in C_{0}^{\infty}\left(D ; \mathbb{R}^{n}\right)} \int_{D} f(e(X+D \phi)) \mathrm{d} x:=h(e(X)),
$$

depending only on $e(X)$. Note that $X \rightarrow h(e(X))=Q(F(X))$ is quasiconvex, hence $h$ is quasiconvex on linear strains and $h \leq f$. By definition, $h \leq Q_{e}(f)$. The proof is finished.

We will use the following theorem concerning the existence and properties of Young measures $[5,19,34]$.

Proposition 2.2. Let $\left(z_{j}\right)$ be a bounded sequence in $L^{1}\left(\Omega ; \mathbb{R}^{s}\right)$. Then there exist a subsequence $\left(z_{j_{k}}\right)$ of $\left(z_{j}\right)$ and a family $\left(\nu_{x}\right)_{x \in \Omega}$ of probability measures on $\mathbb{R}^{s}$, depending measurably on $x \in \Omega$, such that

$$
f\left(z_{j_{k}}\right) \rightarrow \int_{\mathbb{R}^{s}} f(\lambda) \mathrm{d} \nu_{x}(\lambda), \quad \text { in } L^{1}(\Omega)
$$

for every continuous function $f: \mathbb{R}^{s} \rightarrow \mathbb{R}$ such that $\left(f\left(z_{j_{k}}\right)\right)$ is sequentially weakly relatively compact in $L^{1}(\Omega)$.

If the sequence $z_{j}$ is in the form $z_{j}=D u_{j}$, where $\Omega \subset \mathbb{R}^{n}$ is open and bounded, and $\left(u_{j}\right)$ is a bounded sequence in $W^{1, p}\left(\Omega, \mathbb{R}^{N}\right)$ for some $1<p \leq \infty$, then the corresponding Young measure $\nu_{x}$ is called $p$-gradient Young measures (see $[10,19,23]$ ). The Young measure is trivial if $\nu_{x}$ is a Dirac measure for a.e. x. In this case there exists a function $u$ such that $\nu_{x}$ is the Dirac measure at $D u(x)$, and up to a subsequence, $D u_{k} \rightarrow D u$ almost everywhere.

One of the restrictions of $p$-gradient Young measures [19] is that for every quasiconvex function $f: M^{N \times n} \rightarrow$ $\mathbb{R}$, satisfying $|f(X)| \leq C\left(1+|X|^{p}\right)$ when $1<p<\infty$,

$$
\int_{M^{N \times n}} f(\lambda) \mathrm{d} \nu_{x} \geq f\left(\int_{M^{N \times n}} \lambda \mathrm{d} \nu_{x}\right)
$$

for almost every $x \in \Omega$, (see for example, $[8,10,19])$.

For $r>0$ and $x \in \mathbb{R}^{n}$, let $B_{r}(x)=\left\{y \in \mathbb{R}^{n}:|y-x|<r\right\}$ and meas $\left(B_{r}(x)\right)=\omega_{n} r^{n}$, we have ([30]),

Definition 2.3. (The Maximal Function) Let $f \in L_{l o c}^{1}\left(\mathbb{R}^{n}\right)$, we define

$$
(M f)(x)=\sup _{r>0} \frac{1}{\omega_{n} r^{n}} \int_{B_{r}(x)}|f(y)| \mathrm{d} y
$$

where $\omega_{n}$ is the volume of the $n$ dimensional unit ball. 
We have the following weak- $(1,1)$ and strong- $(p, p)$ estimates [30][p. 5, Th. 1(b)]:

Proposition 2.3. If $f \in L^{1}\left(\mathbb{R}^{n}\right)$, then for every $\lambda>0$,

$$
\operatorname{meas}\left(\left\{x \in \mathbb{R}^{n}:(M f)(x)>\lambda\right\}\right) \leq \frac{C(n)}{\lambda} \int_{\mathbb{R}^{n}}|f| \mathrm{d} x .
$$

If $f \in L^{p}\left(\mathbb{R}^{n}\right)$ for $1<p<\infty$, there is a constant $C(n, p)>0$ such that

$$
\|(M f)\|_{L^{p}\left(\mathbb{R}^{n}\right)} \leq C(n)\|f\|_{L^{p}\left(\mathbb{R}^{n}\right)},
$$

which also implies the weak- $(p, p)$ estimate

$$
\operatorname{meas}\left(\left\{x \in \mathbb{R}^{n}:(M f)(x)>\lambda\right\}\right) \leq \frac{C(n, p)}{\lambda^{p}} \int_{\mathbb{R}^{n}}|f|^{p} \mathrm{~d} x .
$$

The following results on convolution operators can be found in ([30], Ch. II., Ths. 3 and 4)

Proposition 2.4. Let $K: \mathbb{R}^{n} \rightarrow \mathbb{R}$ be a 0-homogeneous function, smooth with mean value zero on the unit sphere $S^{n-1}$. Then for $f \in L^{p}\left(\mathbb{R}^{n}\right), 1 \leq p<\infty$, define

$$
T_{\epsilon}(f)(x)=\int_{|y-x| \geq \epsilon} \frac{K(y-x)}{|y-x|^{n}} f(y) \mathrm{d} y, \quad \epsilon>0 . \quad \text { Then }
$$

(a) there exists a constant $A_{p}>0$ independent of $\epsilon>0$ so that

$$
\left\|T_{\epsilon}(f)\right\|_{L^{p}\left(\mathbb{R}^{n}\right)} \leq A_{p}\|f\|_{L^{p}\left(\mathbb{R}^{n}\right)}, \quad 1<p<\infty ;
$$

(b) $\lim _{\epsilon \rightarrow 0} T_{\epsilon}(f)=T(f)$ exists in $L^{p}\left(\mathbb{R}^{n}\right)$ norm $(1<p<\infty)$ while $\lim _{\epsilon \rightarrow 0} T_{\epsilon}(f)(x)$ exists for almost every $x \in \mathbb{R}^{n}$ when $1 \leq p<\infty$ and

$$
\|T(f)\|_{L^{p}\left(\mathbb{R}^{n}\right)} \leq A_{p}\|f\|_{L^{p}\left(\mathbb{R}^{n}\right)}, \quad 1<p<\infty
$$

(c) let $T^{*}(f)(x)=\sup _{\epsilon>0}\left|T_{\epsilon}(f)(x)\right|$. If $f \in L^{1}\left(\mathbb{R}^{n}\right)$, then the mapping $f \rightarrow T^{*}(f)$ is of weak type- $(1,1)$, that is,

$$
\operatorname{meas}\left(\left\{x \in \mathbb{R}^{n}:\left(T^{*} f\right)(x)>\lambda\right\}\right) \leq \frac{C(n)}{\lambda} \int_{\mathbb{R}^{n}}|f| \mathrm{d} x, \quad \text { for all } \lambda>0 ;
$$

(d) if $1<p<\infty$, then $\left\|T^{*}(f)\right\|_{L^{p}\left(\mathbb{R}^{n}\right)} \leq A_{p}\|f\|_{L^{p}\left(\mathbb{R}^{n}\right)}$, which implies the weak-(p,p) estimate

$$
\operatorname{meas}\left(\left\{x \in \mathbb{R}^{n}:\left(T^{*} f\right)(x)>\lambda\right\}\right) \leq \frac{C(n, p)}{\lambda^{p}} \int_{\mathbb{R}^{n}}|f|^{p} \mathrm{~d} x .
$$

The following are some useful estimates for functions in the space of bounded deformations $B D(\Omega)$ and $B D\left(\mathbb{R}^{n}\right) \quad[3,12,21]$. To simplify the statements, we only state the results for functions in $W^{1,1}$ which is contained in $B D$.

Let $\mathcal{R}$ be the class of rigid motions in $\mathbb{R}^{n}$, that is, affine functions of the form $A x+d$ with $A$ skew-symmetric $n \times n$ matrix and $d \in \mathbb{R}^{n}$. The following Poincaré type inequality is in [21] for functions in $B D(\Omega)$, however, we only consider functions in $W^{1,1}\left(\Omega, \mathbb{R}^{n}\right) \subset B D(\Omega)$.

Proposition 2.5. Let $\Omega \subset \mathbb{R}^{n}$ be a bounded, connected open set with Lipschitz boundary and let $R: B D(\Omega) \rightarrow \mathcal{R}$ be a continuous linear mapping which leaves the elements of $\mathcal{R}$ fixed. Then there exists a constant $C(\Omega, R)>0$ such that

$$
\int_{\Omega}|u-R(u)| \mathrm{d} x \leq C(\Omega, R) \int_{\Omega}|e(D u)| \mathrm{d} x, \quad \text { for all } u \in W^{1,1}\left(\Omega, \mathbb{R}^{n}\right) .
$$


When $\Omega \subset \mathbb{R}^{n}$ is an open ball, there is a precise representation of the rigid motion $R(u)$ given by the following result [3] (we still state it for $W^{1,1}$ functions).

Proposition 2.6. Let $u \in W^{1,1}\left(\mathbb{R}^{n}, \mathbb{R}^{n}\right), x \in \mathbb{R}^{n}$ and $\epsilon>0$. Then there exist a vector $d_{\epsilon}(e(D u))(x)$ and a skew-symmetric matrix $A_{\epsilon}(e(D u))(x)$, such that

$$
\int_{B_{\epsilon}(x)}\left|u(y)-A_{\epsilon}(e(D u))(x)(y-x)-d_{\epsilon}(e(D u))(x)\right| \mathrm{d} y \leq C(n) \epsilon \int_{B_{\epsilon}(x)}|e(D u(y))| \mathrm{d} y
$$

where $C(n)>0$ is a constant. Furthermore $d_{\epsilon}(\cdot)$ and $A_{\epsilon}(\cdot)$ can be represented as singular integrals

$$
\begin{aligned}
d_{\epsilon}^{i}(F)(x) & =\sum_{l, m=1}^{n} \int_{|y-x| \geq \epsilon} \frac{\Lambda_{l m}^{i}(y-x)}{n w_{n}|y-x|^{n}} F_{l m}(y) \mathrm{d} y, \\
A_{\epsilon}^{i j}(F)(x) & =\sum_{l, m=1}^{n} \int_{|y-x| \geq \epsilon} \frac{\Gamma_{l m}^{i j}(y-x)}{2 w_{n}|y-x|^{n+2}} F_{l m}(y) \mathrm{d} y,
\end{aligned}
$$

where $F \in L^{p}\left(\mathbb{R}^{n}, M_{s}^{n}\right)$, with $1 \leq p<\infty, \Lambda$ and $\Gamma$ are third and forth order smooth tensors with zero average on $S^{n-1}$, and $\Lambda$ is 0 -homogeneous and $\Gamma$ 2-homogeneous respectively.

If we define $A^{*}(F)(x)=\sup _{\epsilon>0}\left|A_{\epsilon}(F)(x)\right|$, we see from Proposition 2.5 that $A^{*}$ have the weak- $(1,1)$, strong- $(p, p)$ and weak- $(p, p)$ estimates for $F \in L^{p}\left(\mathbb{R}^{n}, M_{s}^{n}\right), 1 \leq p<\infty$.

The following is a simple variation of ([12], Th. 3.1) - the Luzin type theorem for $B D$ functions. In the original statement in [12], it was stated for the case $\lambda=\tau$ with our notation. However, by examining the proof, it is easy to see that the following can be deduced by using the original proof. We also notice that $S_{u}=\emptyset$ in our setting, where $S_{u}$ is the singular part of $u$ (see [3]).

Proposition 2.7. Let $u \in C_{0}^{\infty}\left(\mathbb{R}^{n}, \mathbb{R}^{n}\right)$. We define for any $\lambda>0$ and $\tau>0$

$$
E^{\lambda}=\left\{x \in \mathbb{R}^{n}, M(e(D u))(x)<\lambda\right\}, \quad H^{\tau}=\left\{x \in \mathbb{R}^{n}, A^{*}(e(D u))(x)<\tau\right\},
$$

where $A^{*}$ is defined as above. Then there is a Lipschitz mapping $v_{\lambda, \tau}: \mathbb{R}^{n} \rightarrow \mathbb{R}^{n}$ such that

$$
\left|v_{\lambda, \tau}(x)-v_{\lambda, \tau}(y)\right| \leq C(n)(\lambda+\tau)|x-y|, \quad x, y \in \mathbb{R}^{n}
$$

such that $u(x)=v_{\lambda, \tau}(x)$ on $W_{\lambda, \tau}=E^{\lambda} \cap H^{\tau}$.

We conclude this section by stating a special form of Korn's inequality ([20], Th. 8):

Proposition 2.8. Let $\Omega \subset \mathbb{R}^{n}$ be as in Proposition 2.5 and let $u \in W^{1, p}\left(\Omega, \mathbb{R}^{n}\right), 1<p<\infty$. Then there is a skew-symmetric matrix $A_{u}$ such that

$$
\int_{\Omega}\left|D u(x)-A_{u}\right|^{p} \mathrm{~d} x \leq C \int_{\Omega}|e(D u(x))|^{p} \mathrm{~d} x,
$$

where $C>0$ is a constant independent of $u$ and $A_{u}$.

\section{Proof of Theorem 1 and Corollary 1}

We prove Theorem 1 through Lemma 3.1 to Lemma 3.5.

Proof of Theorem 1. The idea of the proof is the following. We use Lemma 3.1 and Lemma 3.2 to show that $e\left(D u_{j}\right)$ is "essentially" bounded in $L^{\infty}$. Then for a carefully chosen subsequence $u_{j_{k}}$ we use the Luzin type theorem for $B D$ functions to obtain a sequence $\left(v_{k}\right)$ of Lipschitz mappings such that $v_{k}(x)=u_{j_{k}}(x)$ 
on a large subset $W_{j_{k}}$ of $\Omega$ while the Lipschitz constants of $\left(v_{k}\right)$ are unbounded (Lem. 3.3). Then we show (Lem. 3.4) that the linear strains $e\left(D v_{k}\right)$ of these Lipschitz functions are in fact, bounded in $L^{p}$ by using the estimates on $A^{*}$ defined in Proposition 2.6 and the maximal function of $e\left(D u_{j}\right)$. Finally we prove that $\lim _{k \rightarrow \infty} \int_{\Omega}\left|e\left(D u_{j_{k}}-D v_{k}\right)\right|=0$ (Lem. 3.5).

Lemma 3.1. Under the assumptions of Theorem 1 we have

$$
\lim _{j \rightarrow \infty} \int_{\left\{x \in \Omega,\left|e\left(D u_{j}(x)\right)\right| \geq 2 R\right\}}\left|e\left(D u_{j}(x)\right)\right| \mathrm{d} x=0,
$$

and $\left|e\left(D u_{j}(x)\right)\right|$ is equi-integrable on $\Omega$.

Proof. Since $\lim _{j \rightarrow \infty} \int_{\Omega} \operatorname{dist}\left(e\left(D u_{j}(x)\right), \bar{B}_{R}(0)\right) \mathrm{d} x=0$, we see that

$$
\begin{aligned}
0 & =\lim _{j \rightarrow \infty} \int_{\Omega} \operatorname{dist}\left(e\left(D u_{j}(x)\right), \bar{B}_{R}(0)\right) \mathrm{d} x \\
& \geq \limsup _{j \rightarrow \infty} \int_{\left\{x \in \Omega,\left|e\left(D u_{j}(x)\right)\right| \geq 2 R\right\}} \operatorname{dist}\left(e\left(D u_{j}(x)\right), \bar{B}_{R}(0)\right) \mathrm{d} x \\
& \geq \limsup _{j \rightarrow \infty} R \text { meas }\left(\left\{x \in \Omega,\left|e\left(D u_{j}(x)\right)\right| \geq 2 R\right\}\right),
\end{aligned}
$$

where we have used the fact that when $\left|e\left(D u_{j}(x)\right)\right| \geq 2 R$, $\operatorname{dist}\left(e\left(D u_{j}(x)\right), \bar{B}_{R}(0)\right) \geq R$. Next since we have

$$
\operatorname{dist}\left(e\left(D u_{j}(x)\right), \bar{B}_{R}(0)\right) \geq\left|e\left(D u_{j}(x)\right)\right|-R,
$$

whenever $\left|e\left(D u_{j}(x)\right)\right|>R$, we see that

$$
\begin{aligned}
0 & =\lim _{j \rightarrow \infty} \int_{\Omega} \operatorname{dist}\left(e\left(D u_{j}(x)\right), \bar{B}_{R}(0)\right) \mathrm{d} x \\
& \geq \limsup _{j \rightarrow \infty} \int_{\left\{x \in \Omega,\left|e\left(D u_{j}(x)\right)\right| \geq 2 R\right\}} \operatorname{dist}\left(e\left(D u_{j}(x)\right), \bar{B}_{R}(0)\right) \mathrm{d} x \\
& \geq \limsup _{j \rightarrow \infty} \int_{\left\{x \in \Omega,\left|e\left(D u_{j}(x)\right)\right| \geq 2 R\right\}}\left(\left|e\left(D u_{j}(x)\right)\right|-R\right) \mathrm{d} x,
\end{aligned}
$$

hence the first conclusion follows. The second claim is easy to prove because $\bar{B}_{R}(0)$ is compact. In fact, if we let $b_{j}=\int_{\Omega} \operatorname{dist}\left(e\left(D u_{j}(x)\right), \bar{B}_{R}(0)\right) \mathrm{d} x$, then $b_{j} \rightarrow 0$ as $j \rightarrow \infty$. For any measurable subset $G$ of $\Omega$, we have, from (3.2) that

$$
b_{j} \geq \int_{G} \operatorname{dist}\left(e\left(D u_{j}(x)\right), \bar{B}_{R}(0)\right) \mathrm{d} x \geq \int_{G}\left|e\left(D u_{j}(x)\right)\right| \mathrm{d} x-R \operatorname{meas}(G)
$$

so that $\int_{G}\left|e\left(D u_{j}(x)\right)\right| \mathrm{d} x \leq b_{j}+R \operatorname{meas}(G)$. The equi-integrability of $\left|e\left(D u_{j}(x)\right)\right|$ on $\Omega$ then follows easily from this inequality. In fact, for any $\epsilon>0$, if we first choose $\delta_{1}=\epsilon /(2 R)$, then there is some $N>0$, such that $b_{j}<\epsilon / 2$ and $\int_{G}\left|e\left(D u_{j}(x)\right)\right| \mathrm{d} x \leq \epsilon$ whenever $j>N$ and meas $(G)<\delta_{1}$. Let $\delta_{2}>0$ be such that $\int_{G}\left|e\left(D u_{j}(x)\right)\right| \mathrm{d} x \leq \epsilon$ when meas $(G)<\delta_{2}$ and $1 \leq j \leq N$. Now if we take $\delta=\min \left\{\delta_{1}, \delta_{2}\right\}$, the second conclusion then follows.

Now, if

$$
a_{j}=\int_{\left\{x \in \Omega,\left|e\left(D u_{j}(x)\right)\right| \geq 2 R\right\}}\left|e\left(D u_{j}(x)\right)\right| \mathrm{d} x,
$$

we see that $a_{j} \rightarrow 0$ as $j \rightarrow \infty$ which follows from the proof of Lemma 3.1.

Now we extend $u_{j}$ to be defined on $\mathbb{R}^{n}$ by zero, then we may consider the maximal function $M\left(\left|e\left(D u_{j}\right)\right|\right)(x)$ of $\left|e\left(D u_{j}(x)\right)\right|$. We have 
Lemma 3.2. For $\lambda>2 R$, let

$$
E_{j}^{\lambda}=\left\{x \in \mathbb{R}^{n}, M\left(\left|e\left(D u_{j}\right)\right|\right)(x)<\lambda\right\} \text {, hence }\left(E_{j}^{\lambda}\right)^{c}=\left\{x \in \mathbb{R}^{n}, M\left(\left|e\left(D u_{j}\right)\right|\right)(x) \geq \lambda\right\} \text {. }
$$

Let $a_{j}$ be as defined in (3.3), then

$$
\operatorname{meas}\left(\left(E_{j}^{\lambda}\right)^{c}\right) \leq \frac{C(n) a_{j}}{\lambda-2 R} \rightarrow 0 \quad \text { as } \quad j \rightarrow \infty
$$

Proof. The proof of Lemma 3.2 is very similar to that of ([38], Lem. 3.1). We define

$$
h(t)=\left\{\begin{array}{l}
0, \quad \text { if },|t| \leq 2 R \\
|t|-2 R, \quad \text { if },|t| \geq 2 R .
\end{array}\right.
$$

Then $h: \mathbb{R} \rightarrow \mathbb{R}_{+}$is a continuous function. We claim that

$$
\left\{x \in \mathbb{R}^{n}, M\left(\left|e\left(D u_{j}\right)\right|\right)(x) \geq \lambda\right\} \subset\left\{x \in \mathbb{R}^{n}, M\left(h\left(\left|e\left(D u_{j}\right)\right|\right)\right)(x) \geq \lambda-2 R\right\}
$$

We prove (3.5) as follows. If $M\left(\left|e\left(D u_{j}\right)\right|\right)(x) \geq \lambda$, by definition, there is a sequence of positive numbers $\epsilon_{k}>0$ and $r_{k}>0$ with $\epsilon_{k} \rightarrow 0$ as $k \rightarrow \infty$ such that

$$
\frac{1}{\operatorname{meas}\left(B_{r_{k}}(x)\right)} \int_{B_{r_{k}}(x)}\left|e\left(D u_{j}(y)\right)\right| \mathrm{d} y \geq \lambda-\epsilon_{k} .
$$

Since

$$
\begin{aligned}
M\left(h\left(\left|e\left(D u_{j}\right)\right|\right)\right)(x) \geq & \frac{1}{\operatorname{meas}\left(B_{r_{k}}(x)\right)} \int_{B_{r_{k}}(x)} h\left(\left|e\left(D u_{j}(y)\right)\right|\right) \mathrm{d} y \\
= & \frac{1}{\operatorname{meas}\left(B_{r_{k}}(x)\right)} \int_{\left\{y \in B_{r_{k}}(x),\left|e\left(D u_{j}(y)\right)\right| \geq 2 R\right\}}\left(\left|e\left(D u_{j}(y)\right)\right|-2 R\right) \mathrm{d} y \\
= & \frac{1}{\operatorname{meas}\left(B_{r_{k}}(x)\right)} \int_{B_{r_{k}}(x)}\left|e\left(D u_{j}(y)\right)\right| \mathrm{d} y-\frac{1}{\operatorname{meas}\left(B_{r_{k}}(x)\right)} \int_{\left\{y \in B_{r_{k}}(x),\left|e\left(D u_{j}(y)\right)\right| \geq 2 R\right\}} 2 R \mathrm{~d} y \\
& -\frac{1}{\operatorname{meas}\left(B_{r_{k}}(x)\right)} \int_{\left\{y \in B_{r_{k}}(x),\left|e\left(D u_{j}(y)\right)\right|<2 R\right\}}\left|e\left(D u_{j}(y)\right)\right| \mathrm{d} y \geq \lambda-\epsilon_{k}-2 R .
\end{aligned}
$$

Passing to the limit $k \rightarrow \infty$ we obtain $M\left(h\left(\mid e\left(D u_{j}\right)\right) \mid\right)(x) \geq \lambda-2 R$. Thus $(3.5)$ is proved.

Now from the weak- $(1,1)$ estimate of the maximal function (Prop. 2.4), we have

$$
\begin{aligned}
\operatorname{meas}\left(\left\{x \in \mathbb{R}^{n}, M\left(h\left(\left|e\left(D u_{j}\right)\right|\right)\right)(x) \geq \lambda-2 R\right\}\right) & \leq \frac{C(n)}{\lambda-2 R} \int_{\mathbb{R}^{n}} h\left(\left|e\left(D u_{j}(y)\right)\right|\right) \mathrm{d} y \\
& \leq \frac{C(n)}{\lambda-2 R} \int_{\left\{y \in \Omega,\left|e\left(D u_{j}(y)\right)\right| \geq 2 R\right\}}\left|e\left(D u_{j}(y)\right)\right| \mathrm{d} y=\frac{C(n)}{\lambda-2 R} a_{j} \rightarrow 0,
\end{aligned}
$$

as $j \rightarrow \infty$, where $a_{j}$ is defined by (3.3). The proof is finished.

Since the sequence $\left(a_{j}\right)$ defined by $(3.3)$ converges to zero as $j \rightarrow \infty$, we may find a subsequence $\left(a_{j_{k}}\right)$ such that

$$
a_{j_{k}} \leq \mathrm{e}^{-(k+1)}
$$

Recall the operator $A^{*}(e(D u))$ defined following Proposition 2.6. Now we apply Proposition 2.7 to our sequence $u_{j_{k}}$. 
Lemma 3.3. Let $\lambda=4 R$ in Lemma 3.2 and let $\tau_{k}=4 k R$, for $k=1,2, \ldots$ Let

$$
H_{j_{k}}=\left\{x \in \mathbb{R}^{n}, A^{*}\left(e\left(D u_{j_{k}}\right)\right)(x)<\tau_{k}\right\}
$$

Then $u_{j_{k}}$ is a Lipschitz mapping on the set

$$
W_{j_{k}}=E_{j_{k}}^{\lambda} \cap H_{j_{k}}=\left\{x \in \mathbb{R}^{n} M\left(\left|e\left(D u_{j}\right)\right|\right)(x)<\lambda, \quad A^{*}\left(e\left(D u_{j_{k}}\right)\right)(x)<\tau_{k}\right\},
$$

satisfying

$$
\left|u_{j_{k}}(x)-u_{j_{k}}(y)\right| \leq C(n)\left(\lambda+\tau_{k}\right)|x-y|=C(n) 4(1+k) R|x-y| .
$$

From Lemma 3.3 and Kirszbraun's theorem [36], there is a Lipschitz extension $v_{k}$ of $u_{j_{k}}$ to $\mathbb{R}^{n}$ such that

$$
\left|v_{k}(x)-v_{k}(y)\right| \leq C(n) 4(1+k) R|x-y|,
$$

for all $x, y \in \mathbb{R}^{n}$ and

$$
\left|D v_{k}(x)\right| \leq C(n) 4(1+k) R,
$$

for almost every $x \in \mathbb{R}^{n}$ and $D v_{k}(x)=D u_{j_{k}}(x)$ almost everywhere on $W_{j_{k}}$ ([16], Lem. 7.7).

Lemma 3.4. There is a constant $C_{0}>0$ independent of $v_{k}$ such that

$$
\int_{\mathbb{R}^{n}}\left|e\left(D v_{k}(x)\right)\right|^{p} \mathrm{~d} x \leq C_{0}
$$

Proof. For a measurable set $S \subset \mathbb{R}^{n}$, we let

$$
J_{p}(w, S)=\int_{S}|e(D w(x))|^{p} \mathrm{~d} x, \quad 1 \leq p<\infty
$$

as long as the right hand side of (3.11) is finite. We then have

$$
\int_{\mathbb{R}^{n}}\left|e\left(D v_{k}(x)\right)\right|^{p} \mathrm{~d} x=J_{p}\left(v_{k}, \mathbb{R}^{n}\right)=J_{p}\left(v_{k}, W_{j_{k}}\right)+J_{p}\left(v_{k},\left(W_{j_{k}}\right)^{c}\right)
$$

We see that

$$
J_{p}\left(v_{k}, W_{j_{k}}\right)=J_{p}\left(u_{j_{k}}, W_{j_{k}}\right) \leq \lambda^{p} \operatorname{meas}(\Omega)=(4 R)^{p} \operatorname{meas}(\Omega) .
$$

This follows from the fact that $D v_{k}=D u_{j_{k}}$ almost everywhere on $W_{j_{k}}, u_{j_{k}}$ is supported in $\Omega, W_{j_{k}} \subset E_{j_{k}}^{\lambda}$ and $\left|e\left(D u_{j_{k}}(x)\right)\right| \leq M\left(\left|e\left(D u_{j_{k}}\right)\right|\right)(x) \leq \lambda=4 R$ on $E_{j_{k}}^{\lambda}$.

We also have

$$
J_{p}\left(v_{k},\left(W_{j_{k}}\right)^{c}\right)=J_{p}\left(v_{k},\left(H_{j_{k}} \cap E_{j_{k}}^{\lambda}\right)^{c}\right) \leq J_{p}\left(v_{k},\left(H_{j_{k}}\right)^{c} \cup\left(E_{j_{k}}^{\lambda}\right)^{c}\right) \leq J_{p}\left(v_{k},\left(H_{j_{k}}\right)^{c}\right)+J_{p}\left(v_{k},\left(E_{j_{k}}^{\lambda}\right)^{c}\right) .
$$

Notice that $\left|D v_{k}(x)\right| \leq C(n) 4 R(k+1)$ and $\lambda=4 R$, we have

$$
\begin{aligned}
J_{p}\left(v_{k},\left(E_{j_{k}}^{\lambda}\right)^{c}\right) & \leq[C(n) 4 R(k+1)]^{p} \operatorname{meas}\left(\left(E_{j_{k}}^{\lambda}\right)^{c}\right) \leq \frac{C(n, p) R^{p}(k+1)^{p}}{(2 R)^{p}} a_{j_{k}} \\
& \leq \frac{C(n, p) R^{p}(k+1)^{p}}{(2 R)^{p}} \mathrm{e}^{-(k+1)} \leq C(n, p)(k+1)^{p} \mathrm{e}^{-(k+1)} \leq C(n, p) .
\end{aligned}
$$


To estimate $J_{p}\left(v_{k},\left(H_{j_{k}}\right)^{c}\right)$, we write $e\left(D u_{j_{k}}(x)\right)=e_{1}\left(D u_{j_{k}}(x)\right)+e_{2}\left(D u_{j_{k}}(x)\right)$, where

$$
\begin{aligned}
& e_{1}\left(D u_{j_{k}}(x)\right)=\left\{\begin{array}{l}
e\left(D u_{j_{k}}(x)\right), \quad \text { if }\left|e\left(D u_{j_{k}}(x)\right)\right|<4 R, \\
0, \quad \text { if }\left|e\left(D u_{j_{k}}(x)\right)\right| \geq 4 R ;
\end{array}\right. \\
& e_{2}\left(D u_{j_{k}}(x)\right)=\left\{\begin{array}{l}
e\left(D u_{j_{k}}(x)\right), \quad \text { if }\left|e\left(D u_{j_{k}}(x)\right)\right| \geq 4 R, \\
0, \quad \text { if }\left|e\left(D u_{j_{k}}(x)\right)\right|<4 R .
\end{array}\right.
\end{aligned}
$$

Then from

$$
A^{*}\left(e\left(D u_{j_{k}}\right)\right)(x)=A^{*}\left(e_{1}\left(D u_{j_{k}}\right)+e_{2}\left(D u_{j_{k}}\right)\right)(x) \leq A^{*}\left(e_{1}\left(D u_{j_{k}}\right)\right)(x)+A^{*}\left(e_{2}\left(D u_{j_{k}}\right)\right)(x),
$$

we have

$$
\left(H_{j_{k}}\right)^{c} \subset\left\{x \in \mathbb{R}^{n}, A^{*}\left(e_{1}\left(D u_{j_{k}}\right)\right)(x) \geq \tau_{k} / 2\right\} \cup\left\{x \in \mathbb{R}^{n}, A^{*}\left(e_{2}\left(D u_{j_{k}}\right)\right)(x) \geq \tau_{k} / 2\right\} .
$$

Since $D u_{j_{k}}$ is supported in $\Omega$ and $e_{1}\left(D u_{j_{k}}\right)(x)$ is a bounded sequence in $L^{\infty}\left(\mathbb{R}^{n}\right)$, we have from the weak- $(p, p)$ estimate of operator $A^{*}$ (Props. 2.4 and 2.6) that

$$
\begin{aligned}
\operatorname{meas}\left(\left\{x \in \mathbb{R}^{n}, A^{*}\left(e_{1}\left(D u_{j_{k}}\right)\right)(x) \geq \tau_{k} / 2\right\}\right) & \leq \frac{C(n, p)}{\left(\tau_{k}\right)^{p}} \int_{\mathbb{R}^{n}}\left|e_{1}\left(D u_{j_{k}}\right)(y)\right|^{p} \mathrm{~d} y \\
& =\frac{C(n, p)}{(4 R k)^{p}} \int_{\Omega}(4 R)^{p} \mathrm{~d} y \leq \frac{C(n, p)}{k^{p}} \operatorname{meas}(\Omega) .
\end{aligned}
$$

Since $e_{2}\left(D u_{j_{k}}\right)$ is bounded in $L^{1}\left(\mathbb{R}^{n}\right)$ and is also supported in $\Omega$, we apply the weak- $(1,1)$ estimate to operator $A^{*}$ :

$$
\begin{aligned}
\operatorname{meas}\left(\left\{x \in \mathbb{R}^{n}, A^{*}\left(e_{2}\left(D u_{j_{k}}\right)\right)(x) \geq \tau_{k} / 2\right\}\right) & \leq \frac{C(n)}{\tau_{k}} \int_{\mathbb{R}^{n}}\left|e_{2}\left(D u_{j_{k}}(y)\right)\right| \mathrm{d} y \\
= & \frac{C(n)}{4 R k} \int_{\left\{x \in \Omega,\left|e\left(D u_{j_{k}}(y)\right)\right| \geq 4 R\right\}}\left|e\left(D u_{j_{k}}(y)\right)\right| \mathrm{d} y \leq \frac{C(n)}{4 R k} a_{j_{k}} \leq \frac{C(n)}{4 R k \mathrm{e}^{k+1}} .
\end{aligned}
$$

Thus

$$
\operatorname{meas}\left(\left(H_{j_{k}}\right)^{c}\right) \leq \frac{C(n, p)}{k^{p}}+\frac{C(n)}{R k e^{(k+1)}} \rightarrow 0
$$

Consequently,

$$
J_{p}\left(v_{k},\left(H_{j_{k}}\right)^{c}\right) \leq C(n, p) R^{p}(1+k)^{p} \operatorname{meas}\left(\left(H_{j_{k}}\right)^{c}\right) \leq C(n, p)\left[R^{p}\left(\frac{1+k}{k}\right)^{p}+\frac{k^{p-1}}{\mathrm{e}^{k+1}}\right] \leq C(n, p, R),
$$

which follows from the simple facts that $(k+1) / k \leq 2$ for $k=1,2, \ldots$ and $k^{p-1} / \mathrm{e}^{k+1} \rightarrow 0$ as $k \rightarrow \infty$. Finally, we sum up these inequalities to obtain

$$
J_{p}\left(v_{k}, \mathbb{R}^{n}\right)=J_{p}\left(v_{k}, \Omega \cap W_{j_{k}}\right)+J_{p}\left(v_{k},\left(W_{j_{k}}\right)^{c}\right) \leq C(n, p, R):=C_{0} .
$$

The proof is then finished.

Note from (3.4) and (3.13), we have meas $\left(W_{j_{k}}^{c}\right) \rightarrow 0$ as $k \rightarrow \infty$.

Lemma 3.5. Let $v_{k}$ be defined as above, then

$$
\lim _{k \rightarrow \infty} \int_{\Omega}\left|e\left(D v_{k}(x)\right)-e\left(D u_{j_{k}}(x)\right)\right| \mathrm{d} x=0 .
$$


Proof of Lemma 3.5. Since $e\left(D v_{k}(x)\right)=e\left(D u_{j_{k}}(x)\right)$ almost everywhere on $W_{j_{k}}$ defined by (3.8), we have

$$
\begin{aligned}
\int_{\Omega}\left|e\left(D v_{k}(x)\right)-e\left(D u_{j_{k}}(x)\right)\right| \mathrm{d} x & \leq \int_{\left(W_{j_{k}}\right)^{c}}\left|e\left(D v_{k}(x)\right)-e\left(D u_{j_{k}}(x)\right)\right| \mathrm{d} x \\
& \leq J_{1}\left(v_{k},\left(W_{j_{k}}\right)^{c}\right)+J_{1}\left(u_{j_{k}}, \Omega \backslash W_{j_{k}}\right) .
\end{aligned}
$$

Since meas $\left(\left(W_{j_{k}}\right)^{c}\right) \rightarrow 0$ as $j \rightarrow \infty$, and $J_{p}\left(v_{k},\left(W_{j_{k}}\right)^{c}\right)$ is bounded, we have, from Hölder's inequality that

$$
0 \leq J_{1}\left(v_{k},\left(W_{j_{k}}\right)^{c}\right) \leq\left(J_{p}\left(v_{k},\left(W_{j_{k}}\right)^{c}\right)^{1 / p}\left(\operatorname{meas}\left(\left(W_{j_{k}}\right)^{c}\right)\right)^{\frac{p-1}{p}} \rightarrow 0\right.
$$

as $k \rightarrow \infty$.

By Lemma 3.1, $\left|e\left(D u_{j_{k}}(x)\right)\right|$ is equi-integrable on $\Omega$. We see that $J_{1}\left(u_{j_{k}}, \Omega \backslash W_{j_{k}}\right) \rightarrow 0$ as $k \rightarrow \infty$. The conclusion follows.

Remark. Recently, I. Fonseca and the referee both explained to me that Theorem 1 may also be proved by using the general theory of $\mathcal{A}$-quasiconvex functions, in particular, the work by Fonseca and Müller ([15], Cor. 2.18), where a second order operator $\mathcal{A}$ was proposed to treat the linear elastic strain ([15], Ex. 3.10(e)). In fact, for the orthogonal complement $E^{\perp}$ of a general subspace $E \subset M^{N \times n}$ without rank-one matrices, if $K \subset E^{\perp}$ is compact and $\operatorname{dist}\left(P_{E^{\perp}}\left(D u_{j}\right), K\right) \rightarrow 0$ in $L^{1}(\Omega)$, is seems a more promising approach by using the $A$-quasiconvexity method than the one used here. The only problem is to work out algebraically the operator $A$ (see [15] for details).

Proof of Corollary 1. By Korn's inequality (Prop. 2.8), we have

$$
\int_{\Omega}\left|D v_{k}(x)-A_{v_{k}}\right|^{p} \mathrm{~d} x \leq C(n, p) \int_{\Omega}\left|e\left(D v_{k}(x)\right)\right|^{p} \mathrm{~d} x .
$$

Since

$$
w_{k}(x)=v_{k}(x)-\frac{1}{\operatorname{meas}(\Omega)} \int_{\Omega} v_{k}(y) \mathrm{d} y-A_{v_{k}}\left(x-x_{k}\right),
$$

where $x_{k} \in \mathbb{R}^{n}$ is such that $\int_{\Omega} A_{v_{k}}\left(x-x_{k}\right) \mathrm{d} x=0$, we see from Poincaré's inequality and (3.14), we see that $\left(w_{k}\right)$ is bounded in $W^{1, p}\left(\Omega, \mathbb{R}^{n}\right)$. Also because $e\left(D w_{k}(x)\right)=e\left(D v_{k}(x)\right)$ in $\Omega$, the conclusion follows from Lemma 3.5 .

\section{Proof of Theorems 2-4 AND ExAmples}

Proof of Theorem 3. Let us consider the quasiconvex function on linear strains $Q_{e} \operatorname{dist}\left(A, K_{\alpha}\right), A \in M_{s}^{n}$. We first prove that $K:=\left\{A \in M_{s}^{n}, Q_{e} \operatorname{dist}\left(A, K_{\alpha}\right)=0\right\}=K_{\alpha}$. Obviously we have $K_{\alpha} \subset K$. We only need to show that $K \subset K_{\alpha}$. Let $A_{0} \in K$. We have from Proposition 2.1 for quasiconvex envelope on linear strains that there is a sequence $\left(\phi_{j}\right) \subset C_{0}^{\infty}\left(D, \mathbb{R}^{n}\right)$ such that

$$
\lim _{j \rightarrow \infty} \int_{D} \operatorname{dist}\left(A_{0}+e\left(D \phi_{j}(x)\right), K_{\alpha}\right) \mathrm{d} x=0,
$$

where $D \subset \mathbb{R}^{n}$ is the unit cube. Let $K_{\alpha, A_{0}}=\left\{Y-A_{0}, Y \in K_{\alpha}\right\}$, then $K_{\alpha, A_{0}}$ is still compact and $\lim _{j \rightarrow \infty} \int_{D} \operatorname{dist}\left(e\left(D \phi_{j}(x)\right), K_{\alpha, A_{0}}\right) \mathrm{d} x=0$. We then have, from Lemma 3.1 that $\left|e\left(D \phi_{j}\right)\right|$ is equi-integrable on $D$, hence up to a subsequence (still denoted by the same subscripts) $e\left(D \phi_{j}\right)$ converges weakly in $L^{1}(D)$. For each fixed $j$, we extend $\phi_{j}$ to be defined in $\mathbb{R}^{n}$ as a periodic function and then let

$$
u_{j}(x)=\frac{1}{j} \phi_{j}(j x)
$$


for $j=1,2, \ldots$ and for $x \in D$. We see that $u_{j} \in C_{0}^{\infty}\left(D, \mathbb{R}^{n}\right)$. Furthermore, $\left|e\left(D u_{j}\right)\right|$ is equi-integrable in $\Omega$, up to a subsequence $e\left(D u_{j}\right) \rightarrow 0$ in $L^{1}\left(D, M_{s}^{n}\right)$ as $j \rightarrow 0$ and

$$
\lim _{j \rightarrow \infty} \int_{D} \operatorname{dist}\left(e\left(D u_{j}(x)\right), K_{\alpha, A_{0}}\right) \mathrm{d} x=0 .
$$

The properties of $u_{j}$ mentioned above should be well-known and can be verified easily. However, for the convenience of the reader, we state them in the following slightly general way (Lem. 4.1 below) and give a proof in the Appendix. Note that we also have $\int_{D} e\left(D \phi_{j}\right) \mathrm{d} x=0$, hence we only need to prove the claims for each component of $e\left(D \phi_{j}\right)$.

Lemma 4.1. Suppose $f_{j} \rightarrow f$ in $L^{1}(D)$ as $j \rightarrow \infty$ and $\int_{D} f_{j} \mathrm{~d} x=0$, where $D \subset \mathbb{R}^{n}$ is the (closed) unit cube. Then if we extend $f_{j}$ to $\mathbb{R}^{n}$ periodically and let $u_{j}(x)=f_{j}(j x), x \in D$, then $\left(u_{j}\right)$ is equi-integrable on $D$, and up to a subsequence $u_{j} \rightarrow 0$ in $L^{1}(D)$ as $j \rightarrow 0$. Furthermore, for any continuous function $W: \mathbb{R} \rightarrow \mathbb{R}$ with linear growth, that is, $|W(t)| \leq C(|t|+1)$, we have $\int_{D} W\left(f_{j}\right) \mathrm{d} x=\int_{D} W\left(u_{j}\right) \mathrm{d} x$.

Proof of Theorem 3 (continued). Now, $\operatorname{since} \lim _{j \rightarrow \infty} \int_{D} \operatorname{dist}\left(e\left(D u_{j}(x)\right), K_{\alpha, A_{0}}\right) \mathrm{d} x=0$, we see, from Theorem 1 and Corollary 1 that there is a subsequence $\left(u_{j_{k}}\right)$ of $\left(u_{j}\right)$ and a bounded sequence $\left(w_{k}\right)$ in $W^{1,2 p}\left(D, \mathbb{R}^{n}\right)$ (hence $\left|D w_{k}\right|^{p}$ is equi-integrable on $\Omega$ ) such that $\left\|e\left(D u_{j_{k}}\right)-e\left(D w_{k}\right)\right\|_{L^{1}(D)} \rightarrow 0$ as $k \rightarrow \infty$. We assert that $e\left(D w_{k}\right) \rightarrow 0$ in $L^{p}$ as $k \rightarrow \infty$. If we let $\nu_{x}$ be the family of Young measures corresponding to $D w_{k}$, we have

$$
0=\int_{M^{n \times n}} e(\tau) \mathrm{d} \nu_{x}(\tau)=e\left(\int_{M^{n \times n}} \tau \mathrm{d} \nu_{x}(\tau)\right),
$$

which follows from the Young measure representation of the weak limit and the fact that $e(\cdot)$ is a linear mapping on $M^{n \times n}$. We also have $\int_{M^{n \times n}} \operatorname{dist}\left(e(\tau), K_{\alpha, A_{0}}\right) \mathrm{d} \nu_{x}(\tau)=0$ for almost every $x \in \Omega$. Hence on the support of $\nu_{x}$, $A_{0}+e(\tau) \in K_{\alpha}, \nu_{x}$-almost everywhere. Let $F^{(\alpha)}(Y)=\max \{F(Y)-\alpha, 0\}$, then $F^{(\alpha)}(\cdot)$ is still a quasiconvex function on linear strains with $p$-th growth at infinity, while $\left|e\left(D w_{k}\right)\right|^{p}$ is equi-integrable on $D$, therefore by the lower semicontinuity theorem [1] and the Young measure representation, we have, up to a subsequence,

$$
\begin{aligned}
0 & =\int_{D} \int_{M^{n \times n}} F^{\alpha}\left(A_{0}+e(\tau)\right) \mathrm{d} \nu_{x}(\tau) \mathrm{d} x=\lim _{k \rightarrow \infty} \int_{D} F^{\alpha}\left(A_{0}+e\left(w_{k}(x)\right)\right) \mathrm{d} x \\
& \geq \int_{D} F^{\alpha}\left(A_{0}+e\left(\int_{M^{n \times n}} \tau \mathrm{d} \nu_{x}\right)\right) \mathrm{d} x=\int_{D} F^{\alpha}\left(A_{0}\right) \mathrm{d} x \geq 0 .
\end{aligned}
$$

Hence $F^{\alpha}\left(A_{0}\right)=0$ and $A_{0} \in K_{\alpha}$.

Finally we show that the quasiconvex function on linear strains $Q_{e} \operatorname{dist}^{p}\left(\cdot, K_{\alpha}\right)$ satisfies the requirements (1.5) and (1.6). We observe that for $Y \in M_{s}^{n}$,

$$
\left(Q_{e} \operatorname{dist}\left(Y, K_{\alpha}\right)\right)^{p} \leq Q_{e} \operatorname{dist}^{p}\left(Y, K_{\alpha}\right) .
$$

In fact, let $\phi_{j} \in C_{0}^{\infty}\left(D, \mathbb{R}^{n}\right)$ be a minimizing sequence such that

$$
Q_{e} \operatorname{dist}^{p}\left(e(Y), K_{\alpha}\right)=\lim _{j \rightarrow \infty} \int_{D} \operatorname{dist}^{p}\left(Y+e\left(D \phi_{j}\right), K_{\alpha}\right) \mathrm{d} x,
$$

where $D$ is the unit cube in $\mathbb{R}^{n}$. For each $j>0$, we apply Hölder's inequality to obtain

$$
\begin{aligned}
Q_{e} \operatorname{dist}\left(Y, K_{\alpha}\right) & \leq \int_{D} Q_{e} \operatorname{dist}\left(Y+e\left(D \phi_{j}\right), K_{\alpha}\right) \mathrm{d} x \\
& \leq \int_{D} \operatorname{dist}\left(Y+e\left(D \phi_{j}\right), K_{\alpha}\right) \mathrm{d} x \leq\left(\int_{D} \operatorname{dist}^{p}\left(Y+e\left(D \phi_{j}\right), K_{\alpha}\right) \mathrm{d} x\right)^{1 / p} .
\end{aligned}
$$


Hence (4.1) follows by letting $j \rightarrow \infty$. We see that the zero set of $Q_{e} \operatorname{dist}^{p}\left(Y, K_{\alpha}\right)$ is contained in that of $Q_{e} \operatorname{dist}\left(Y, K_{\alpha}\right)$ which is $K_{\alpha}$. On the other hand, it is obvious that the zero set of $Q \operatorname{dist}^{p}\left(Y, K_{\alpha}\right)$ contains $K_{\alpha}$. The conclusion then follows.

Proof of Theorem 4. Since by definition, we have $\mathbb{Q}_{p}^{e}(K) \subset Q_{p}^{e}(K) \subset Q_{1}^{e}(K)$, we only need to prove that $Q_{1}^{e}(K) \subset \mathbb{Q}_{p}^{e}(K)$ for every $1 \leq p<\infty$.

Let $f: M_{s}^{n} \rightarrow \mathbb{R}$ be quasiconvex on linear strains and $0 \leq f(Y) \leq C_{f}\left(1+|Y|^{p}\right)$ for $Y \in M_{s}^{n}$. We let $\alpha=\max _{Y \in K} f(Y)$ and define $K_{\alpha}=\left\{Y \in M_{s}^{n}, f(Y) \leq \alpha\right\}$, then we see that $\mathbb{Q}_{p}^{e}(K)$ is the intersection of all such $K_{\alpha}$. From Theorem 2, we see that

$$
K_{\alpha}=\left\{Y \in M_{s}^{n}, Q_{e} \operatorname{dist}\left(Y, K_{\alpha}\right)=0\right\} .
$$

On the other hand, since it is obviously true that $K \subset K_{\alpha}$, we have $\operatorname{dist}\left(Y, K_{\alpha}\right) \leq \operatorname{dist}(Y, K)$ for all $Y \in M_{s}^{n}$ so that $0 \leq Q_{e} \operatorname{dist}\left(Y, K_{\alpha}\right) \leq Q_{e} \operatorname{dist}(Y, K)$ hence $Q_{1}^{e}(K) \subset K_{\alpha}$ and $Q_{1}^{e}(K) \subset \mathbb{Q}_{p}^{e}(K)$. The proof is finished.

The following lemma will be used in the proof of Theorem 2 .

Lemma 4.2. Suppose $F: M_{s}^{n} \rightarrow \mathbb{R}$ is continuous and non-negative with $F^{-1}(0)=K$ a non-empty and compact set. Furthermore, assume that $F$ satisfies the coercivity condition $\lim _{|A| \rightarrow \infty} F(A) /|A|=+\infty$. Let $\left(Q_{e}(F)\right)^{-1}(0)=\left\{A \in M_{s}^{n}, Q_{e}(f(A))=0\right\}$. Then $\left(Q_{e}(F)\right)^{-1}(0) \subset Q_{1}^{e}(K)$.

If we take Theorem 4 into account, clearly, Lemma 4.1 also implies $\left(Q_{e}(F)\right)^{-1}(0) \subset \mathbb{Q}_{p}^{e}(K)$ for all $1 \leq p<\infty$.

Proof of Lemma 4.2. Let $A \in\left(Q_{e}(F)\right)^{-1}(0)$, then there is a sequence $\phi_{j} \in C_{0}^{\infty}\left(D, \mathbb{R}^{n}\right)$ such that $\lim _{j \rightarrow \infty} \int_{D} F\left(A+e\left(D \phi_{j}\right)\right) \mathrm{d} x=0$. It is then easy to see, from the coercivity condition that up to a subsequence, $\operatorname{dist}\left(A+e\left(D \phi_{j}\right), K\right) \rightarrow 0$ almost everywhere and $e\left(D \phi_{j}\right)$ is equi-integrable. Hence $\int_{D} \operatorname{dist}\left(A+e\left(D \phi_{j}\right), K\right) \mathrm{d} x \rightarrow 0$ as $j \rightarrow \infty$. By definition, $A \in Q_{1}^{e}(K)$, the proof is finished.

With the help of Theorems 3-5, we now prove Theorem 2.

Proof of Theorem 2. Since $C(f) \leq Q_{e}(f) \leq R_{e}(f)$, we only need to show that if $R_{e}(f) \neq C f$ then $Q_{e}(f) \neq C f$.

For $f: M_{s}^{n} \rightarrow \mathbb{R}$, let epi $(f)=\left\{(A, t) \in M_{s}^{n} \times \mathbb{R}, f(A) \geq t\right\}$ be the epi-graph of $f$. First we claim that there is a supporting plane $E$ (see [29]) of epi $(C f)$ in $M_{s}^{n}$ such that $K_{0}=\operatorname{epi}\left(R_{e} f\right) \cap E$ is not convex while $C K_{0}=\operatorname{epi}(C(f)) \cap E$. If this is not true, we can easily see that $R_{e}(f)=C(f)$ on $K=\mathcal{P}(\mathcal{K})=,\mathcal{P}(\mathcal{C}(\mathcal{K})$,$) ,$ where $K=\mathcal{P}\left(\mathcal{K}_{\prime}\right)$ is the orthogonal projection of $K_{0} \subset M_{s}^{n} \times \mathbb{R}$ to $M_{s}^{n}$, so that $R_{e}(f) \equiv C(f)$ (see [29]), and we reach a contradiction.

Now, we use the supporting plane $E$ to construct a non-negative rank-one convex function $F$ on linear strains, vanishing exactly on $K$ with superlinear growth (1.5) so that $K$ is also compact.

Since the plane $E$ is the graph of a real-valued affine function $L(\cdot)$ defined on $M_{s}^{n}$, we see that $R_{e}(f(\cdot))-L(\cdot) \geq$ 0 and $R_{e}(f(A))-L(A)=0$ if and only if $A \in K$. We also see that $K$ is compact because $R_{e}(f) \geq C f$ hence $R_{e}(f)$ satisfies

$$
\lim _{|A| \rightarrow \infty} \frac{R_{e}(f(A))}{|A|}=+\infty .
$$

Let us consider $F(A)=R_{e}(f(A))-L(A)$, for $A \in M_{s}^{n}$. Then $X \rightarrow F(e(X))$ is rank-one convex, $F \geq 0$, and $F(A)=0$ if and only if $A \in K$, and

$$
\lim _{|A| \rightarrow \infty} \frac{F(A)}{|A|}=+\infty
$$

Next we show that $Q_{e}(f(P))>C f(P)$ for a certain matrix $P \in M_{s}^{n}$, if $Q_{e}(F(P))>0$ holds.

From Proposition 2.1 and the fact that $L$ is affine, we see that

$$
Q_{e}(F(\cdot))=Q_{e}\left[R_{e}(f(\cdot))-L(\cdot)\right]=Q_{e}(f(\cdot))-L(\cdot) .
$$


Therefore, we only need to prove that $Q_{e}(F)$ is not convex. Since $F \geq 0$, it suffices to show that the zero set of $Q_{e}(F)$

is not convex.

$$
Q_{e} F^{-1}(0)=\left\{A \in M_{s}^{n}, Q_{e}(F(A))=0\right\}
$$

From Theorem 5, we see that $\mathbb{Q}_{2}^{e}(K)$ is not convex because the non-convex set $K$ is the zero set of the non-negative rank-one convex function $F$ on linear strains, so it is a closed lamination convex set. We also notice that $C(f(\cdot))-L(\cdot) \geq 0$ and $C(f(A))-L(A)=0$ if and only if $A \in C(K)$.

Let $P \in C(K) \backslash \mathbb{Q}_{2}^{e}(K)$. From Theorem 4 , we see that $\mathbb{Q}_{2}^{e}(K)=\mathbb{Q}_{1}^{e}(K)=Q_{1}^{e}(K)$. From Lemma 4.2 we see further that $\left(Q_{e}(F)\right)^{-1}(0) \subset Q_{1}^{e}(K)$. Thus $Q_{e}(F(P))>0$ which implies

$$
Q_{e}(F(P))=Q_{e}(f(P))-L(P)>0=C f(P)-L(P)
$$

and $Q_{e}(f(P))>C f(P)$. The proof is finished.

Now let us examine some examples of quasiconvex functions defined on linear strains with linear growth.

Example 4.1. Let $I \in M^{n \times n}$ be the unit matrix. It was proved in [13] following an argument in [31], that for

$$
K_{0}=\{-I, I\} \subset M^{n \times n},
$$

$Q \operatorname{dist}\left(e(X), K_{0}\right)$ does not vanish at $X=0$, hence $Q \operatorname{dist}\left(e(X), K_{0}\right)$ is not convex. In fact, it was established earlier in [41] that for any closed set $K \subset E_{\partial}$ - the subspace of conformal matrices (or $K \subset E_{\bar{\partial}}$ - the subspace of anti-conformal matrices) of $M^{2 \times 2}$, there is a constant $c>0$ independent of $K$ such that

$$
Q \operatorname{dist}(X, K) \geq c \operatorname{dist}(X, K)
$$

for all $X \in M^{2 \times 2}$. This result was generalized in [17] to the case when $K \subset E$ where $E \subset M^{N \times n}$ is a subspace without rank-one matrices, that is

$$
Q \operatorname{dist}(X, K) \geq c(E) \operatorname{dist}(X, K)
$$

where $c(E)>0$ depends only on $E$. Therefore the following much improved estimate of the above result in [13] for $K_{0}$ can be deduced from these earlier results:

$$
Q_{e} \operatorname{dist}\left(Y, K_{0}\right) \geq c(n) \operatorname{dist}\left(Y, K_{0}\right) .
$$

In the $2 \times 2$ case we observe that $K_{0} \times\left(M_{s}^{2}\right)^{\perp} \subset E_{\partial}$, thus from (4.4), we have

$$
Q_{e} \operatorname{dist}\left(e(X), K_{0}\right)=Q \operatorname{dist}\left(X, K_{0} \times\left(M_{s}^{2}\right)^{\perp}\right) \geq c \operatorname{dist}\left(X, K_{0} \times\left(M_{s}^{2}\right)^{\perp}\right)=c \operatorname{dist}\left(e(X), K_{0}\right) .
$$

For the $n \times n$ case, since it is known $[10,22]$ that the subspace $E=\operatorname{span}\{I\} \oplus\left(M_{s}^{n}\right)^{\perp}$ does not have rank-one matrices, so, we see from (4.4) that (4.5) holds if we replace $E_{\partial}$ by $E$.

Example 4.2. From a special case of the explicit calculation of the quasiconvex relaxation for the two linear strain energy [22], we see that the quasiconvex function on linear strains $Q_{e} \operatorname{dist}^{2}\left(Y, K_{0}\right)$ satisfies that

$$
Q_{e} \operatorname{dist}^{2}\left(Y, K_{0}\right)=\operatorname{dist}^{2}\left(Y, K_{0}\right)
$$

when $\operatorname{dist}^{2}\left(Y, K_{0}\right)$ is small, where $K_{0}=\{-I, I\} \subset M_{s}^{n}$ is as defined in Example 4.1. We see that the sublevel set

$$
K_{\alpha^{2}}=\left\{Y \in M_{s}^{n}, Q_{e} \operatorname{dist}^{2}\left(e(Y), K_{0}\right) \leq \alpha^{2}\right\}=\bar{B}_{\alpha}(I) \cup \bar{B}_{\alpha}(-I)
$$

when $\alpha>0$ is small, where $\bar{B}_{\alpha}(I)$ and $\bar{B}_{\alpha}(-I)$ are closed balls in $M_{s}^{n}$ centered at $I$ and $-I$ respectively with radius $\alpha$. We may also make the two closed balls disjoint. We see that $K_{\alpha^{2}}=Q_{1}^{e}\left(K_{\alpha^{2}}\right)$ hence the zero set of the following quasiconvex function on linear strains with linear growth $Q_{e} \operatorname{dist}\left(Y, K_{\alpha^{2}}\right)$ is $K_{\alpha^{2}}$ itself. 
Acknowledgements. This work is partially supported by the Australian Research Council through the ARC Research Grant Scheme. I would like to thank Irene Fonseca and the referee for helpful suggestions.

\section{APPENDiX}

Proof of Lemma 4.1. Since $f_{j} \rightarrow f$ in $L^{1}(D)$, we have, from Dunford-Pettis Theorem [14], $\left|f_{j}\right|$ is equi-integrable on $D$. Thus for every $\epsilon>0$, there is some $\delta>0$, such that $\int_{E}\left|f_{j}\right| \mathrm{d} x \leq \epsilon$ for all $j>0$ whenever $E \subset D$ is measurable and meas $(E) \leq \delta$. We let $j E-m=\{j x-m, x \in E\}$ for $m \in \mathbb{R}^{n}$. Then we can decompose $j D$ into $j^{n}$ unit cubes $\left(D_{s}\right)$ whose vertex that is closest to the origin $m_{s}$, - a vector with integer components between 0 and $j^{n}-1$. Also the intersection between different $D_{s}$ 's is only on the boundary. We have $j D=\cup_{s=1}^{j^{n}} D_{s}$. Thus, since $f_{j}$ is now periodic with period $D$,

$$
\begin{aligned}
\int_{E}\left|u_{j}(x)\right| \mathrm{d} x & =\int_{E}\left|f_{j}(j x)\right| \mathrm{d} x=\frac{1}{j^{n}} \int_{j E}\left|f_{j}(y)\right| \mathrm{d} y \\
& =\frac{1}{j^{n}} \sum_{s=1}^{j^{n}} \int_{j E \cap D_{s}}\left|f_{j}(y)\right| \mathrm{d} y=\frac{1}{j^{n}} \sum_{s=1}^{j^{n}} \int_{\left(j E \cap D_{s}\right)-m_{s}}\left|f_{j}(y)\right| \mathrm{d} y .
\end{aligned}
$$

Since meas $(E) \leq \delta, \operatorname{meas}(j E) \leq j^{n} \delta, \sum_{s=1}^{j^{n}} \operatorname{meas}\left[\left(j E \cap D_{s}\right)-m_{s}\right]=\operatorname{meas}(j E) \leq j^{n} \delta$ and $\left(j E \cap D_{s}\right)-m_{s} \subset D$. We may write, for each $s$, meas $\left[\left(j E \cap D_{s}\right)-m_{s}\right]=j_{s} \delta+r_{s} \delta$ where $j_{s} \geq 0$ is an integer and $0 \leq r_{s}<1$. Thus

$$
\sum_{s=1}^{j^{n}} j_{s} \delta \leq \sum_{s=1}^{j^{n}}\left(j_{s} \delta+r_{s} \delta\right) \leq j^{n} \delta
$$

so that $\sum_{s=1}^{j^{n}} j_{s} \leq j^{n}$. We also have meas $\left[\left(j E \cap D_{s}\right)-m_{s}\right] \leq\left(j_{s}+1\right) \delta$, hence it is easy to prove that $\int_{\left(j E \cap D_{s}\right)-m_{s}}\left|f_{j}(y)\right| \mathrm{d} y \leq\left(j_{s}+1\right) \epsilon$, which implies

$$
\frac{1}{j^{n}} \sum_{s=1}^{j^{n}} \int_{\left(j E \cap D_{s}\right)-m_{s}}\left|f_{j}(y)\right| \mathrm{d} y \leq 2 \epsilon
$$

Therefore $\left|u_{j}\right|$ is equi-integrable on $D$.

Again from Dunford-Pettis Theorem, up to a subsequence $u_{j} \rightarrow u$ in $L^{1}(D)$ for some $u \in L^{1}(D)$. We only need to show that $u=0$ almost everywhere. Since we have assumed that $D$ is closed, for each $\phi \in C_{0}(D)$, we consider

$$
\int_{D} u_{j} \phi \mathrm{d} x=\int_{D} f_{j}(j x) \phi(x) \mathrm{d} x=\frac{1}{j^{n}} \int_{j D} f_{j}(y) \phi\left(\frac{y}{j}\right) \mathrm{d} y=\int_{D} f_{j}(y)\left(\sum_{s=1}^{j^{n}} \phi\left(\frac{y}{j}-m_{s}\right) \frac{1}{j^{n}}\right) \mathrm{d} y .
$$

Then, from the boundedness of $f_{j}$ in $L^{1}(D)$, the assumption $\int_{D} f_{j} \mathrm{~d} x=0$ and the definition of Riemann integral, we see that $\int_{D} u_{j} \phi \mathrm{d} x \rightarrow 0$ as $j \rightarrow \infty$ hence $u=0$.

The last claim that $\int_{D} W\left(u_{j}(x)\right) \mathrm{d} x=\int_{D} W\left(f_{j}(x)\right) \mathrm{d} x$ can be easily checked by changing the variable $j x=y$ and the periodicity assumption on $f_{j}$.

The following is the proof of Theorem 5 extracted from [44]. We need some preparations.

A quadratic function $q: M_{s}^{n} \rightarrow \mathbb{R}$ is called a rank-one convex quadratic function on linear strains if $X \rightarrow$ $q\left(e(X)\right.$ is a rank-one convex quadratic function defined on $M^{n \times n}$. We denote by $R C_{e}$ the set of all rank-one convex quadratic functions on linear strains. 
Definition A.1. The quadratic rank-one convex hull $q r^{e}(K)$ of a compact set $K \subset M_{s}^{n}$ is defined by

$$
q r^{e}(K)=\left\{X \in M_{s}^{n}, q(X) \leq \sup _{Y \in K} q(Y), q \in R C_{e}\right\}
$$

From the definition of $q r^{e}(K)$, one can easily see that

$$
K \subset L_{c}^{e}(K) \subset \mathbb{R}_{2}^{e}(K) \leq \mathbb{Q}_{2}^{e}(K) \subset q r^{e}(K) \subset C(K)
$$

Therefore if we can prove that $q r^{e}(K)=C(K)$ if and only if $L_{c}^{e}(K)=C(K)$, Theorem 5 will then follows.

Let $E \subset M_{s}^{n}$ be a linear subspace without compatible matrices, and $E^{\perp}$ being its orthogonal complement of $E$ in $M_{s}^{n}$. Let

$$
q(A)=\left|P_{E^{\perp}}(A)\right|^{2}-\lambda_{E}\left|P_{E}(A)\right|^{2},
$$

where $P_{E^{\perp}}$ and $P_{E}$ are orthogonal projections to $E^{\perp}$ and $E$ respectively, and $\lambda_{E}>0$ is the largest positive number such that the quadratic form $q$ is a rank-one convex (so is quasiconvex) on linear strains. The constant $\lambda_{E}$ can be defined as follows. Since $E$ does not have compatible strains, $E \oplus\left(M_{s}^{n}\right)^{\perp}$ does not have rank-one matrices. Note that $P_{E^{\perp}}(a \otimes b)=P_{E^{\perp}}(e(a \otimes b))$ and $\left|P_{E^{\perp}}(a \otimes b)\right|^{2}>0$ for any nonzero rank-one matrix $a \otimes b$. Let $a, b \in \mathbb{R}^{n}$, we then define

$$
\frac{1}{\lambda_{E}}=\sup _{|a|=|b|=1} \frac{\left|P_{E}(e(a \otimes b))\right|^{2}}{\left|P_{E^{\perp}}(e(a \otimes b))\right|^{2}}<+\infty
$$

and $\lambda_{E}>0$ satisfies the requirement.

If $E_{1}$ is a plane in $M_{s}^{n}$ parallel to $E$ and $X \in E_{1}$, then

$$
q_{X}(A)=\left|P_{E^{\perp}}(A)\right|^{2}-\lambda_{E}\left|P_{E}(A-X)\right|^{2}
$$

is a quadratic rank-one convex function reaching its strict maximum at $X$ in $E_{1}$ with $q_{X}(X)=0$ and $q_{X}(A)<0$ for $A \in E_{1} \backslash\{X\}$. We have

Lemma A.1. Suppose $E \subset M_{s}^{n}$ is a linear subspace without compatible matrices and $E_{1}$ is a plane parallel to $E$. Then any closed subset $K \subset E_{1}$ is a quadratic rank-one convex set of linear strains, that is, qre $(K)=K$.

Proof. If $K \neq E_{1}$, then for any $X \in E_{1} \backslash K$, we consider $q_{X}$ defined by (A.3), then $q_{X} \in R C_{e}$ and $q_{X}(X)=$ $0>\sup _{A \in K} q_{X}(A)$. Therefore $X \notin q r^{e}(K)$. The proof is then finished.

Proof of Theorem 5. We first show that if $E_{1}$ is a supporting plane (see [29]) of $C(K)$ then

$$
q r^{e}(K) \cap E_{1}=q r^{e}\left(K \cap E_{1}\right)
$$

Let $E$ be the plane in $M_{s}^{n}$ containing $C(K)$ with the same dimension as $C(K)$ (see [29]). Obviously, $q r^{e}(K \cap$ $\left.E_{1}\right) \subset q r^{e}(K) \cap E_{1}$. Let $X \in q r^{e}(K) \cap E_{1}$. There is an affine function $l$ defined on $M_{s}^{n}$ such that $l<0$ on the open half space in $E$ containing $C(K) \backslash E_{1}, l=0$ on $E_{1}$ and $l>0$ on the opposite half space to $C(K)$ in $E$. We also define $E_{1}(\epsilon)=\left\{A \in E\right.$, $\left.\operatorname{dist}\left(A, E_{1}\right) \leq \epsilon, l(A) \leq 0\right\}$ which is a set on the same side as $C(K)$ in $E$, where $\operatorname{dist}\left(A, E_{1}\right)$ is the euclidean distance from $A$ to $E_{1}$. For any fixed $q \in R C_{e}$ we consider, for every integer $n>0$ the quadratic function $q(\cdot)+n l(\cdot) \in R C_{e}$. Since for any $A \in K \cap E_{1}, l(A)=0$, we have, for every fixed point $X \in q r^{e}(K) \cap E_{1}$,

$$
q(X)=q(X)+n l(X) \leq \sup _{A \in K}[q(A)+n l(A)]
$$

Since $q+n l$ is continuous and $K$ compact, the maximum is attained at some $A_{n} \in K$, that is, $\sup _{A \in K}[q(A)+$ $n l(A)]=q\left(A_{n}\right)+n l\left(A_{n}\right)$, so that $q(X) \leq q\left(A_{n}\right)+n l\left(A_{n}\right)$. Since $K$ is compact there is a subsequence $A_{n_{k}} \rightarrow$ 
$A_{0} \in K$ as $k \rightarrow 0$. Notice that $l\left(A_{n}\right) \leq 0$ for all $n$. If we let $k \rightarrow \infty$ we see that $\delta_{k}:=\operatorname{dist}\left(A_{n_{k}}, E_{1}\right) \rightarrow 0$. Otherwise $q(X)$ cannot be finite. Now we have

$$
q(X) \leq q\left(A_{n_{k}}\right)+n_{k} l\left(A_{n_{k}}\right) \leq \sup \left\{q(A), A \in K \cap E_{1}\left(\delta_{k}\right)\right\} .
$$

Again the "sup" in (A.5) can be reached by, say $B_{k} \in K \cap E_{1}\left(\delta_{l}\right)$, and up to a subsequence $B_{k} \rightarrow B_{0} \in K \cap E_{1}$ as $k \rightarrow \infty$.

Passing to the limit $k \rightarrow 0$ on both side of the inequality $q(X) \leq q\left(B_{k}\right)$ and noticing that $B_{0} \in K \cap E_{1}$, we have $q(X) \leq q\left(B_{0}\right) \leq \sup _{A \in K \cap E_{1}} q(A)$, hence $X \in q r^{e}\left(K \cap E_{1}\right),\left(\right.$ A.4) is proved. Notice also that $C(K) \cap E_{1}=$ $C\left(K \cap E_{1}\right)$.

Now suppose $K \subset M_{s}^{n}$ is compact while $L_{c}^{e}(K) \neq C(K)$, but $q r^{e}(K)=C(K)$. We may assume that $K$ is a closed laminated convex set. Then among all these $K$ 's there is one for which the affine dimension $\operatorname{dim} C(K) \geq 1$ of $C(K)$ is the smallest. For such $K$ we claim that the plane $E$ in $M_{s}^{n}$ spanned by $C(K)$ does not have compatible pairs. Otherwise it is easy to see that there is a supporting plane $E_{1}$ of $C(K)$ such that $E_{1} \cap K$ is still a closed laminated convex set on linear strains while $q r^{e}\left(K \cap E_{1}\right)=q r^{e}(K) \cap E_{1}=C(K) \cap E_{1}$ is convex. This contradicts to the fact that the dimension $\operatorname{dim} C(K)$ is the smallest. Now since $C(K) \subset E$ and $E$ does not have compatible pairs, there is some $X \in C(K) \neq K$. If we define $q_{X}$ as in Lemma A.1, then there is $\delta>0$, such that $q_{X}(X)=0>-\delta=\sup _{A \in K \subset E} q(A)$. Hence $X \notin q r^{e}(K)$ and $q r^{e}(K) \neq C(K)$, a contradiction.

\section{REFERENCES}

[1] E. Acerbi and N. Fusco, Semicontinuity problems in the calculus of variations. Arch. Ration. Mech. Anal. 86 (1984) 125-145.

[2] R.A. Adams, Sobolev Spaces. Academic Press (1975).

[3] L. Ambrosio, A. Coscia and G. Dal Maso, Fine properties of functions with bounded deformation. Arch. Ration. Mech. Anal. 139 (1997) 201-238.

[4] J.M. Ball, Convexity conditions and existence theorems in nonlinear elasticity. Arch. Ration. Mech. Anal. 63 (1977) $337-403$.

[5] J.M. Ball, A version of the fundamental theorem of Young measures, in Partial Differential Equations and Continuum Models of Phase Transitions, M. Rascle, D. Serre and M. Slemrod Eds., Springer-Verlag (1989) 207-215.

[6] J.M. Ball and R.D. James, Fine phase mixtures as minimizers of energy. Arch. Ration. Mech. Anal. 100 (1987) 13-52.

[7] J.M. Ball and R.D. James, Proposed experimental tests of a theory of fine microstructures and the two-well problem. Phil. R. Soc. Lond. Sect. A 338 (1992) 389-450.

[8] J.M. Ball and K. Zhang, Lower semicontinuity of multiple integrals and the biting lemma. Proc. R. Soc. Edinb. Sect. A 114 (1990) 367-379.

[9] K. Bhattacharya, Comparison of the geometrically nonlinear and linear theories of martensitic transformation. Continuum Mech. Thermodyn. 5 (1993) 205-242.

[10] K. Bhattacharya, N.B. Firoozy, R.D. James and R.V. Kohn, Restrictions on Microstructures. Proc. R. Soc. Edinb. Sect. A 124 (1994) 843-878.

[11] B. Dacorogna, Direct Methods in the Calculus of Variations. Springer (1989).

[12] F.B. Ebobisse, Luzin-type approximation of BD functions. Proc. R. Soc. Edin. Sect. A 129 (1999) $697-705$.

[13] F.B. Ebobisse, On lower semicontinuity of integral functionals in $L D(\Omega)$. Preprint Univ. Pisa.

[14] I. Ekeland and R. Temam, Convex Analysis and Variational Problems. North-Holland (1976).

[15] I. Fonseca and S. Müller, A-quasiconvexity, lower semicontinuity and Young measures. SIAM J. Math. Anal. 30 (1999) $1355-1390$.

[16] D. Gilbarg and N. Trudinger, Elliptic Partial Differential Equations of Second Order. Second edn, Academic Press (1983).

[17] Z. Iqbal, Variational Methods in Solid Mechanics. Ph.D. thesis, University of Oxford (1999).

[18] A.G. Khachaturyan, Theory of Structural Transformations in Solids. John Wiley and Sons (1983).

[19] D. Kinderlehrer and P. Pedregal, Characterizations of Young measures generated by gradients. Arch. Ration. Mech. Anal. 115 (1991) 329-365.

[20] V.A. Kondratev and O.A. Oleinik, Boundary-value problems for the system of elasticity theory in unbounded domains. Russian Math. Survey 43 (1988) 65-119.

[21] R.V. Kohn, New estimates for deformations in terms of their strains. Ph.D. thesis, Princeton University (1979).

[22] R.V. Kohn, The relaxation of a double-well energy. Cont. Mech. Therm. 3 (1991) 981-1000.

[23] J. Kristensen, Lower semicontinuity in spaces of weakly differentiable functions. J. Math. Ann. 313 (1999) 653-710.

[24] K. de Leeuw and H. Mirkil, Majorations dans $L_{\infty}$ des opérateurs différentiels à coefficients constants. C. R. Acad. Sci. Paris 254 (1962) 2286-2288.

[25] F.C. Liu, A Luzin type property of Sobolev functions. Ind. Univ. Math. J. 26 (1977) 645-651. 
[26] C.B. Jr Morrey, Multiple integrals in the calculus of variations. Springer (1966).

[27] S. Müller, A sharp version of Zhang's theorem on truncating sequences of gradients. Trans. AMS 351 (1999) $4585-4597$.

[28] S. Müller and V. Šverák, Attainment results for the two-well problem by convex integration, in Geometric analysis and the calculus of variations, Internat. Press, Cambridge, MA (1996) 239-251.

[29] R.T. Rockafellar, Convex Analysis. Princeton University Press (1970).

[30] E.M. Stein, Singular Integrals and Differentiability Properties of Functions. Princeton University Press, Princeton (1970).

[31] V. Šverák, Quasiconvex functions with subquadratic growth. Proc. R. Soc. Lond. Sect. A 433 (1991) 723-725.

[32] V. Šverák, Rank one convexity does not imply quasiconvexity. Proc. R. Soc. Edinb. Sect. A 120 (1992) 185-189.

[33] V. Šverák, On the problem of two wells in Microstructure and Phase Transition. IMA Vol. Math. Appl. 54 (1994) 183-189.

[34] L. Tartar, Compensated compactness and applications to partial differential equations, in Nonlinear Analysis and Mechanics: Heriot-Watt Symp., R.J. Knops Ed., IV (1979) 136-212.

[35] R. Temam, Problèmes Mathématiques en Plasticité. Gauthier-Villars (1983).

[36] J.H. Wells and L.R. Williams, Embeddings and extensions in analysis. Springer-Verlag (1975).

[37] B.-S. Yan, On $W^{1, p_{-}}$quasiconvex hulls of set of matrices. Preprint.

[38] K.-W. Zhang, A construction of quasiconvex functions with linear growth at infinity. Ann. Sc. Norm Sup. Pisa. Serie IV XIX (1992) 313-326.

[39] K.-W. Zhang, Quasiconvex functions, $S O(n)$ and two elastic wells. Anal. Nonlin. H. Poincaré 14 (1997) 759-785.

[40] K.-W. Zhang, On the structure of quasiconvex hulls. Anal. Nonlin. H. Poincaré 15 (1998) 663-686.

[41] K.-W. Zhang, On some quasiconvex functions with linear growth. J. Convex Anal. 5 (1988) 133-146.

[42] K.-W. Zhang, Rank-one connections at infinity and quasiconvex hulls. J. Convex Anal. 7 (2000) 19-45.

[43] K.-W. Zhang, On some semiconvex envelopes in the calculus of variations. NoDEA - Nonlinear Diff. Equ. Appl. 9 (2002) $37-44$

[44] K.-W. Zhang, On equality of relaxations for linear elastic strains. Commun. Pure Appl. Anal. 1 (2002) 565-573. 